\title{
Internal transport barriers in the National Spherical Torus Experiment ${ }^{a}$
}

\author{
H. Y. Yuh, ${ }^{1, b)}$ F. M. Levinton, ${ }^{1}$ R. E. Bell, ${ }^{2}$ J. C. Hosea, ${ }^{2}$ S. M. Kaye, ${ }^{2}$ B. P. LeBlanc, ${ }^{2}$ \\ E. Mazzucato, ${ }^{2}$ J. L. Peterson, ${ }^{2}$ D. R. Smith, ${ }^{2}$ J. Candy, ${ }^{3}$ R. E. Waltz, ${ }^{3}$ C. W. Domier, ${ }^{4}$ \\ N. C. Luhmann, Jr., ${ }^{4}$ W. Lee, ${ }^{5}$ and H. K. Park ${ }^{5}$ \\ ${ }^{1}$ Nova Photonics Inc., Princeton, New Jersey 08540, USA \\ ${ }^{2}$ Princeton Plasma Physics Laboratory, Princeton University, Princeton, New Jersey 08543, USA \\ ${ }^{3}$ General Atomics, San Diego, California 92186-5608, USA \\ ${ }^{4}$ University of California at Davis, Davis, California 95616, USA \\ ${ }^{5}$ POSTECH, Pohang 790-784, Korea
}

(Received 7 December 2008; accepted 14 April 2009; published online 20 May 2009)

\begin{abstract}
In the National Spherical Torus Experiment [M. Ono et al., Nucl. Fusion 41, 1435 (2001)], internal transport barriers (ITBs) are observed in reversed (negative) shear discharges where diffusivities for electron and ion thermal channels and momentum are reduced. While neutral beam heating can produce ITBs in both electron and ion channels, high harmonic fast wave heating can also produce electron ITBs (e-ITBs) under reversed magnetic shear conditions without momentum input. Interestingly, the location of the e-ITB does not necessarily match that of the ion ITB (i-ITB). The e-ITB location correlates best with the magnetic shear minima location determined by motional Stark effect constrained equilibria, whereas the i-ITB location better correlates with the location of maximum $\mathbf{E} \times \mathbf{B}$ shearing rate. Measured electron temperature gradients in the e-ITB can exceed critical gradients for the onset of electron thermal gradient microinstabilities calculated by linear gyrokinetic codes. A high- $k$ microwave scattering diagnostic shows locally reduced density fluctuations at wave numbers characteristic of electron turbulence for discharges with strongly negative magnetic shear versus weakly negative or positive magnetic shear. Reductions in fluctuation amplitude are found to be correlated with the local value of magnetic shear. These results are consistent with nonlinear gyrokinetic simulations predicting a reduction in electron turbulence under negative magnetic shear conditions despite exceeding critical gradients. (C) 2009 American Institute of Physics. [DOI: 10.1063/1.3129163]
\end{abstract}

\section{INTRODUCTION}

Understanding the underlying mechanisms that lead to improved plasma confinement in internal transport barriers (ITBs) has been a continuous topic of interest in magnetic fusion research, because ITBs have the potential to become a powerful tool much like the $H$-mode edge transport barriers. Improved confinement resulting from ITBs has been previously reported on many tokamaks, with a multimachine review found in Ref. 1 and also more recently in a spherical torus. ${ }^{2}$ Here we report on measurements of ITBs in the National Spherical Torus Experiment (NSTX), where improved confinement for reversed shear plasmas is observed simultaneously in the electron and ion thermal channels, as well as in toroidal momentum. Calculated thermal diffusivities inside the steep gradient region of the ITBs can reach similar values on NSTX as found on devices operating at much higher magnetic fields.

Due to the large momentum input from neutral beam injection (NBI), ion transport on NSTX can be reduced to neoclassical values, consistent with the existing understanding that ion temperature gradient (ITG) microinstability induced transport can be quenched with sufficient $\mathbf{E} \times \mathbf{B}$ flow shear. However, this stabilization does not seem to also effectively suppress transport in the electron channel, resulting

\footnotetext{
a) Paper TI2 5, Bull. Am. Phys. Soc. 53, 241 (2008).

${ }^{b)}$ Invited speaker. Electronic mail: hyuh@pppl.gov.
}

in a dominant loss channel through the electrons. In the current work, we explore a new regime of electron confinement where electron temperature profiles are no longer stiff at sufficiently negative magnetic shear. After a general description of the plasmas used in this work, we begin by examining the kinetic profiles of the ITBs followed by a statistical analysis of key profile features. Local electron scale fluctuations inside the ITBs will be examined and the experimental results will be compared with gyrokinetic simulations.

\section{PLASMA DESCRIPTION}

Plasmas discussed in this work were all performed at $0.55 \mathrm{~T}$, with $1 \mathrm{MA}$ of plasma current, using a combination of NBI and high harmonic fast wave (HHFW) radio frequency heating. ${ }^{3,4}$ The $L$-mode discharges can display a large range of shear reversal, or negative magnetic shear $s \equiv(r / q) d q / d r$, during the early portion of the discharge. Figure 1 shows the time evolution of such an ITB discharge, in which an early period of reversed shear, indicated by the elevated $q_{0^{-}} q_{\mathrm{min}}$, accompanies a rapid rise in the core electron and ion temperatures, until MHD activity causes the current to redistribute on a timescale much faster than the current relaxation time. The $q$ and toroidal current profiles during and after the ITB phase are shown in Fig. 2. The plasma most often continues with a weakly reversed shear or monotonic $q$-profile, during which the electron confinement 


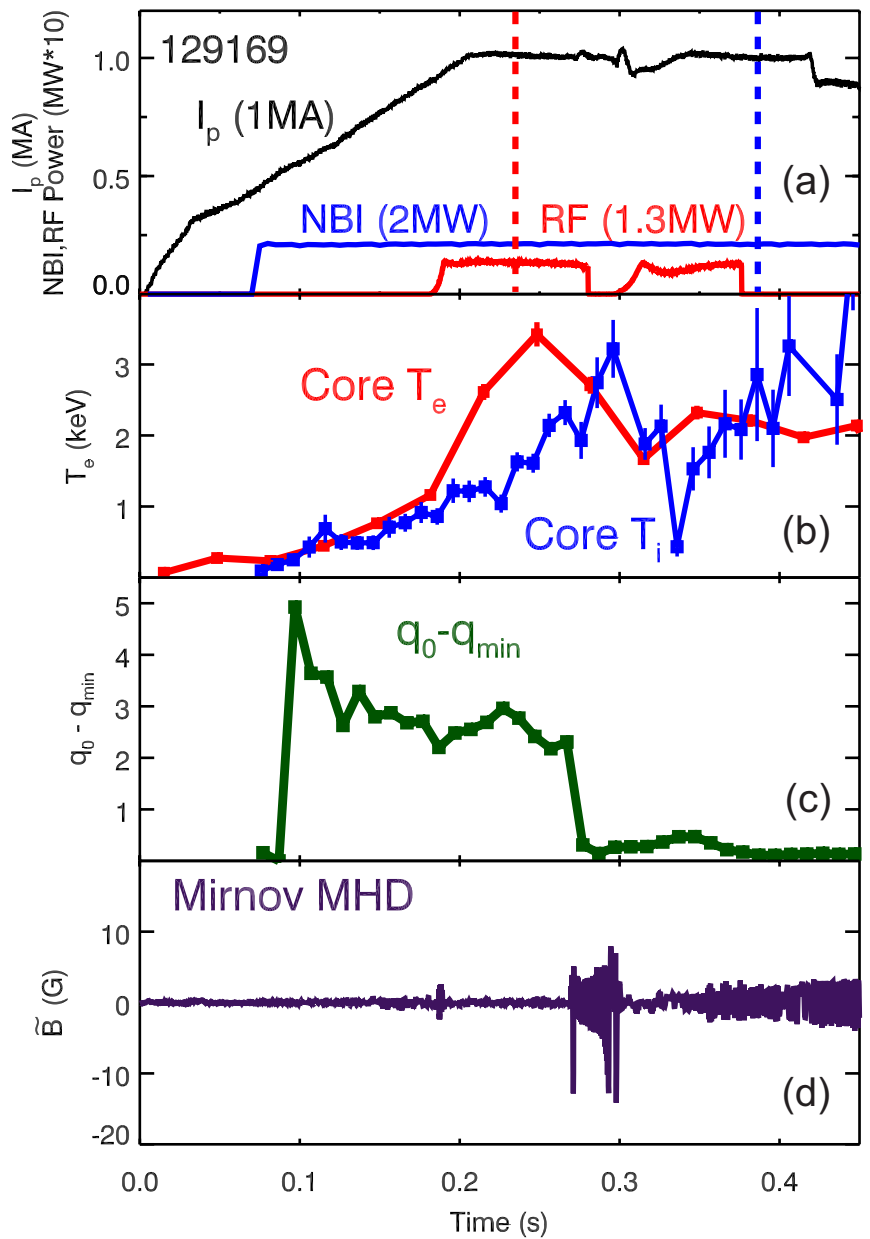

FIG. 1. (Color online) Time evolution of an initially strongly reversed shear plasma displaying an e-ITB until the current redistribution event shown by the MHD trace. (a) Plasma current and heating waveforms, (b) core electron and ion temperatures, (c) elevated $q_{0}-q_{\min }$ indicates reversed shear conditions, and (d) MHD activity. Dashed lines in (a) correspond to times in Fig. 2.

is degraded relative to the shear reversal period, but the ion confinement does not necessarily degrade as significantly.

\section{PROFILE MEASUREMENTS}

A number of diagnostics on NSTX allow for detailed profile measurements. Shown using the left hand axis in Fig. 3 are kinetic profiles from the same plasma as in Fig. 1. The electron temperature and density, $T_{e}$ and $n_{e}$, are measured using a 30 channel, $60 \mathrm{~Hz}$ Thomson scattering system. ${ }^{5}$ Ion temperature $T_{i}$ and toroidal velocity $v_{\phi}$ are measured using the 51 channel charge exchange recombination spectroscopy (CHERS) (Ref. 6) with $10 \mathrm{~ms}$ time resolution. The $q$-profile is reconstructed using LRDFIT, a free boundary GradShafranov equilibrium reconstruction code that models vacuum vessel eddy currents and makes use of magnetics, electron temperature isothermal flux surfaces, rotation, and $E_{r}$ corrected pitch angles from a 16 channel motional Stark effect (MSE) (Ref. 7) system with $10 \mathrm{~ms}$ time resolution.

Normalized inverse gradient scale lengths, $R / L_{X} \equiv-(R / X)(d X / d r)$, where $R$ is the major radius, are calculated from $\chi^{2}$ minimized fits to the profile data with spline
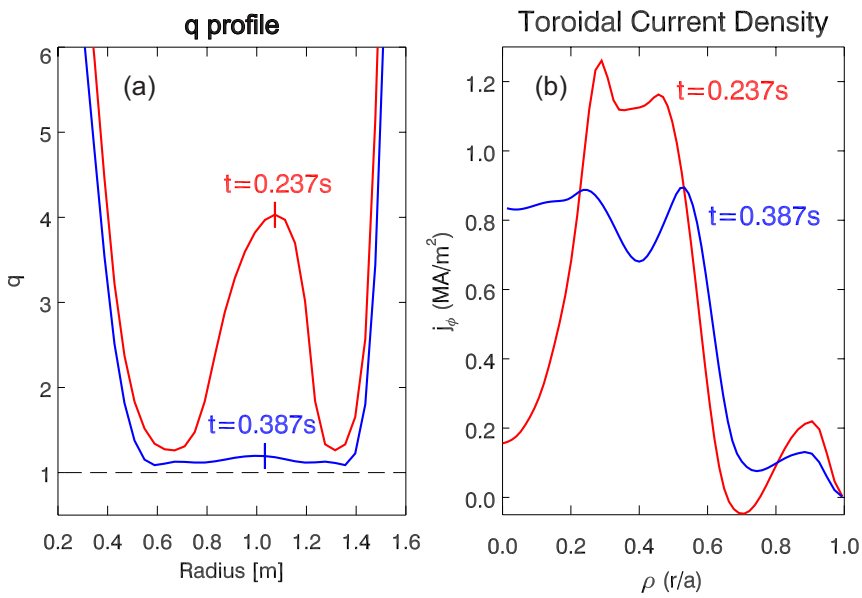

FIG. 2. (Color online) (a) $q$ and (b) current density profiles during and after the reversed shear period.

or tanh functions. Figure 3 shows the normalized inverse gradient scale lengths using the right hand axis. ITB profiles show peaked gradients in the core plasma in the electron and ion temperatures and also in toroidal velocity, while the electron density gradient does not show a dramatic increase with ITB formation. Note that for negative shear $q$-profiles $s_{\min }$, the profile magnetic shear minima are negative quantities, while monotonic $q$-profiles have $s_{\min }=0$. Figure 3 demonstrates that peak gradients do not necessarily occur at the same radial location.

To separate the electron and ion thermal transport channels and subsequently discuss the differences between transport barrier locations for each channel, it is necessary that the ion-electron coupling be weak relative to the thermal transport in each individual channel. This is generally true for the ITB discharges discussed here, where due to the low densities, the ion-electron power exchange is about an order of magnitude below the power transported in either ion or electron channels.

Comparing the location of profile features requires radial alignment cross calibration between the profile instruments. In addition to extensive invessel spatial calibrations, CHERS and Thomson scattering radial alignment has been checked using high density $H$-mode pedestals, where the alignment of the edge pedestal position is observed to agree to within a centimeter. ${ }^{8}$ The MSE system in turn shares an optical system with the CHERS diagnostic and therefore no systematic offsets are expected between these diagnostics.

\section{A. $q_{\min }$ and rational $q$ values}

Statistical analysis was carried out for a database of over 500 kinetic profiles from over 80 discharges for the value and location of profile features. We begin with a discussion of the relationship between $q_{\min }$ and the ITBs.

The location of $s_{\min }$ and the ITBs, defined as the radius of peak $R / L_{T_{e}}(\mathrm{e}-\mathrm{ITB})$ and $R / L_{T_{i}}$ (i-ITB) appears on average $9 \mathrm{~cm}$ away from $q_{\min }$, shown as histograms of the occurrence fraction within the database in Fig. 4. The $q$ values at the ITBs do not favor any particular rational value of $q$ and 


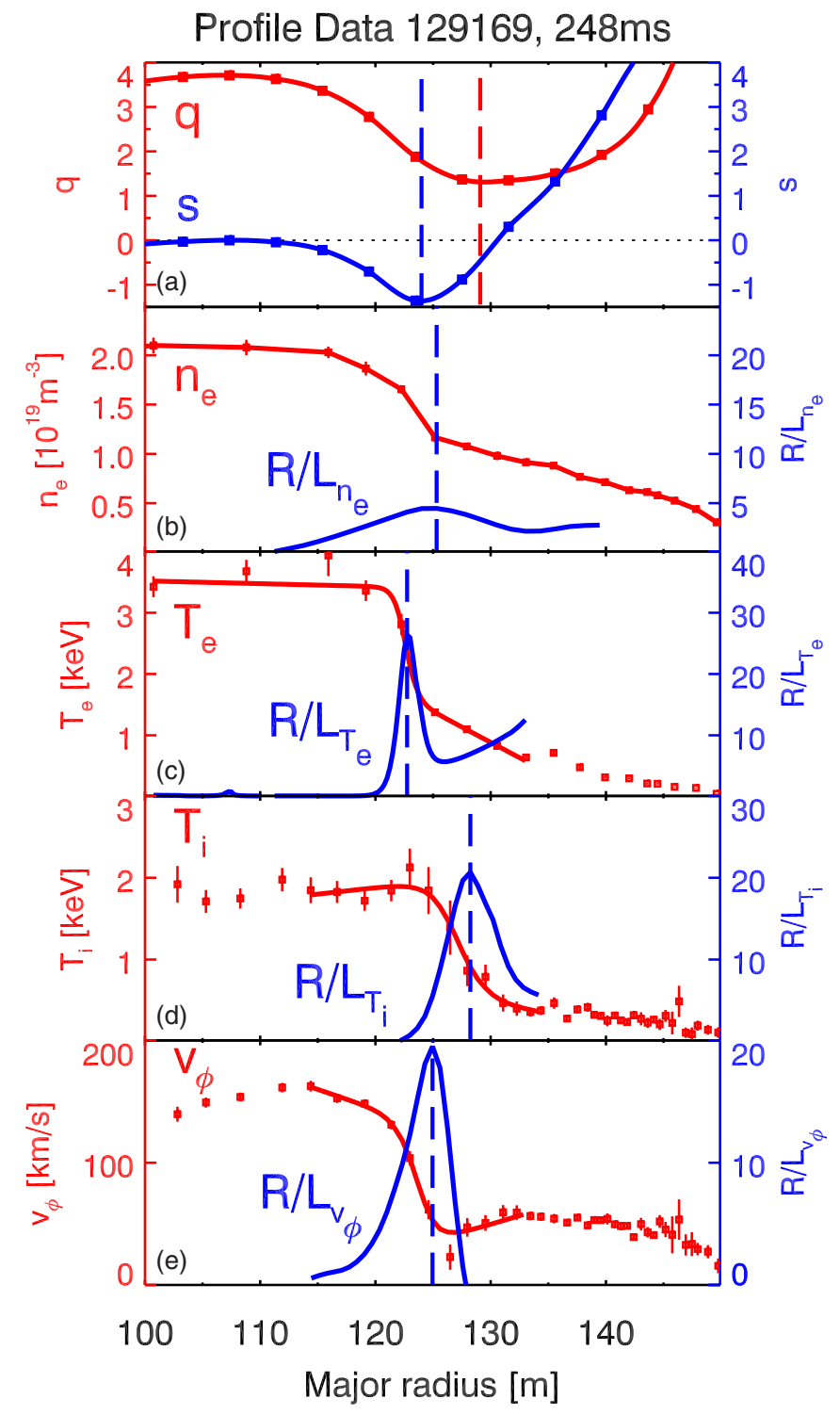

FIG. 3. (Color online) Kinetic profiles of an ITB. Using the left hand axes are (a) $q$ (b) electron density, $n_{e}$ (c) electron temperature, $T_{e}$ (d) ion temperature $T_{i}$, and (e) toroidal velocity $v_{\phi}$. Using the right hand axes are (a) magnetic shear, and [(b)-(e)] the normalized inverse gradient scale lengths. Vertical dashed lines indicate radial positions of interest for $q_{\min }, s_{\min }$, and peak normalized inverse gradient scale lengths.

occur over a range, as seen in Fig. 5. The lack of correlation to rational $q$ values at the transport barriers and the large spatial separation between $q_{\min }$ and the ITBs suggests that these are not key factors for NSTX ITBs.

\section{ION TRANSPORT BARRIER}

Ion transport in i-ITBs appears consistent with the general understanding that ITG microturbulence induced transport can be suppressed with sufficient $\mathbf{E} \times \mathbf{B}$ shear. ${ }^{9-13}$ Figure 6 shows a trend of increasing peak $R / L_{T_{i}}$ with increasing peak $R / L_{v_{\phi}}$, as the toroidal velocity typically provides the largest contribution in the radial force balance equation,

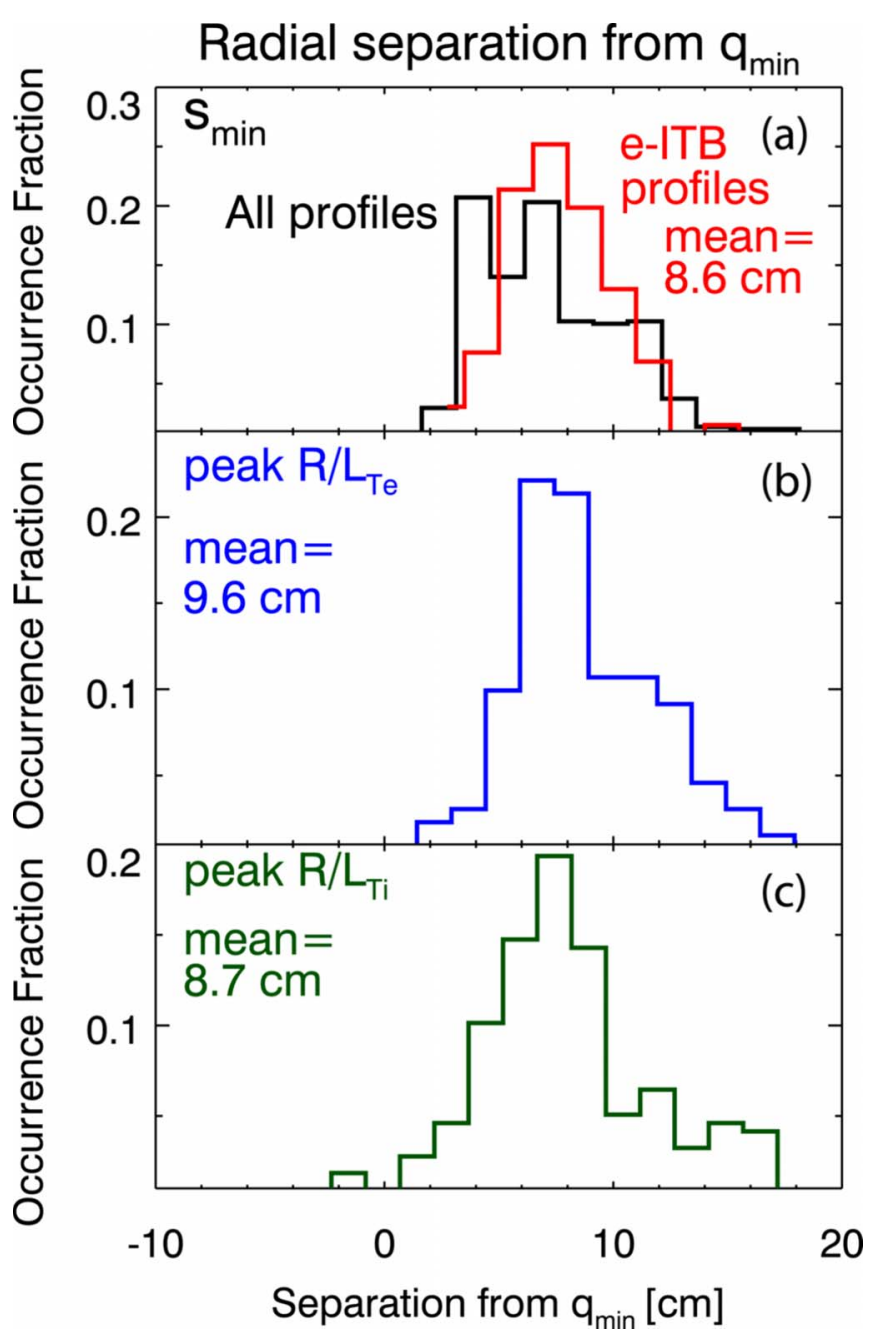

FIG. 4. (Color online) Distribution of radial separation between $q_{\min }$ and (a) $s_{\min }$, for all profiles and e-ITB profiles, (b) the e-ITB (c) the i-ITB. Positive separations indicate that $q_{\min }$ occurs radially further outboard.

$$
E_{r}=\frac{1}{Z_{i} e n_{i}} \nabla P_{i}-v_{\theta i} B_{\phi}+v_{\phi i} B_{\theta}
$$

where $Z_{i} e, n_{i}$, and $P_{i}$ are the ion charge, density, and pressure, and $v$ and $B$ are the velocity and magnetic field in the toroidal $(\phi)$ and poloidal $(\theta)$ directions. The large scatter shows that despite stabilization of ion gyroscale modes, the ITG is coupled to other factors such as electron transport.

The location of the i-ITB is also well correlated with the location of maximum $\mathbf{E} \times \mathbf{B}$ shear. Figure 7 shows that the i-ITB statistically occurs most frequently within approximately $3 \mathrm{~cm}$ of the peak $\mathbf{E} \times \mathbf{B}$ shear position, but with a higher tendency for the i-ITB to occur outboard from the location of peak $\mathbf{E} \times \mathbf{B}$. The reason for this assymmetry is not clear. For the calculation of the $\mathbf{E} \times \mathbf{B}$ shearing rate, the poloidal velocity contribution was neglected due to the lack of calibrated poloidal velocity measurements. This should not effect the results due to the fact that poloidal flows and their gradients are expected to be small in the core, based on neoclassical calculations from NCLASS. ${ }^{14}$ Linear gyrokinetic simulations ${ }^{15}$ show ion gyroscale modes to be stable in ITB profiles. 


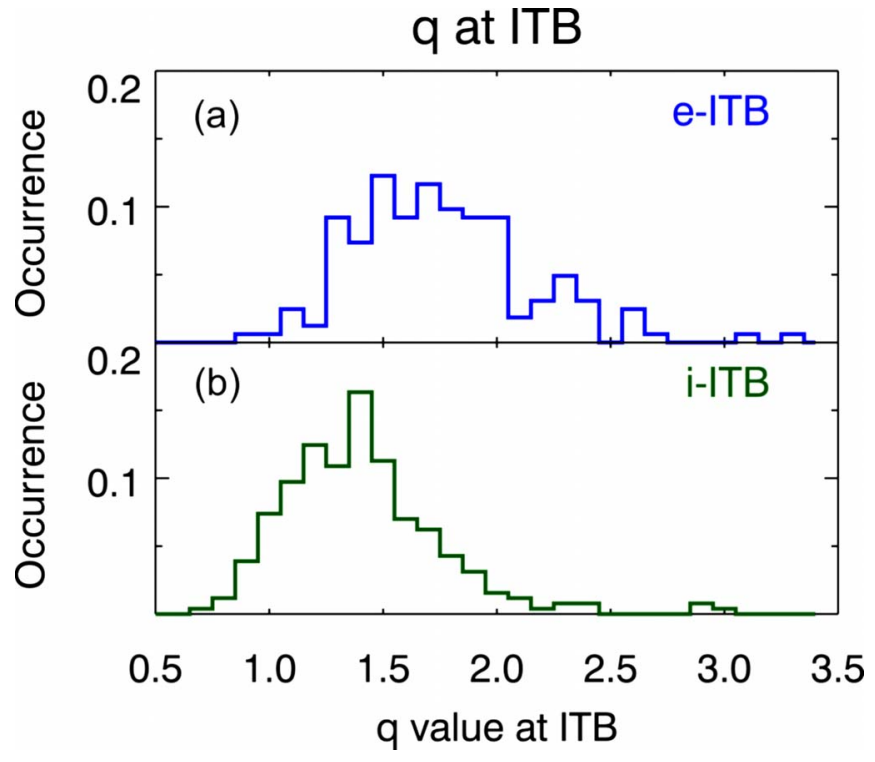

FIG. 5. (Color online) Distribution of $q$ values at (a) the e-ITBs, (b) the i-ITBs.

\section{ELECTRON TRANSPORT BARRIER}

From the profiles shown in Fig. 3, one can see that the e-ITB does not necessarily occur at the i-ITB location. The location of the e-ITB best correlates with the location of minimum magnetic shear. Figure 8 shows the radius of the e-ITBs versus the radius of $s_{\min }$ and a histogram of their separation distance. The e-ITB occurs almost exclusively at $s_{\min }$ or a few centimeters inboard. Overlaid in the same figure, one can see the separation between the i-ITB and $s_{\min }$ is in the opposite direction, with the i-ITB occurring mostly outboard of the $s_{\min }$ location, and better correlated with the

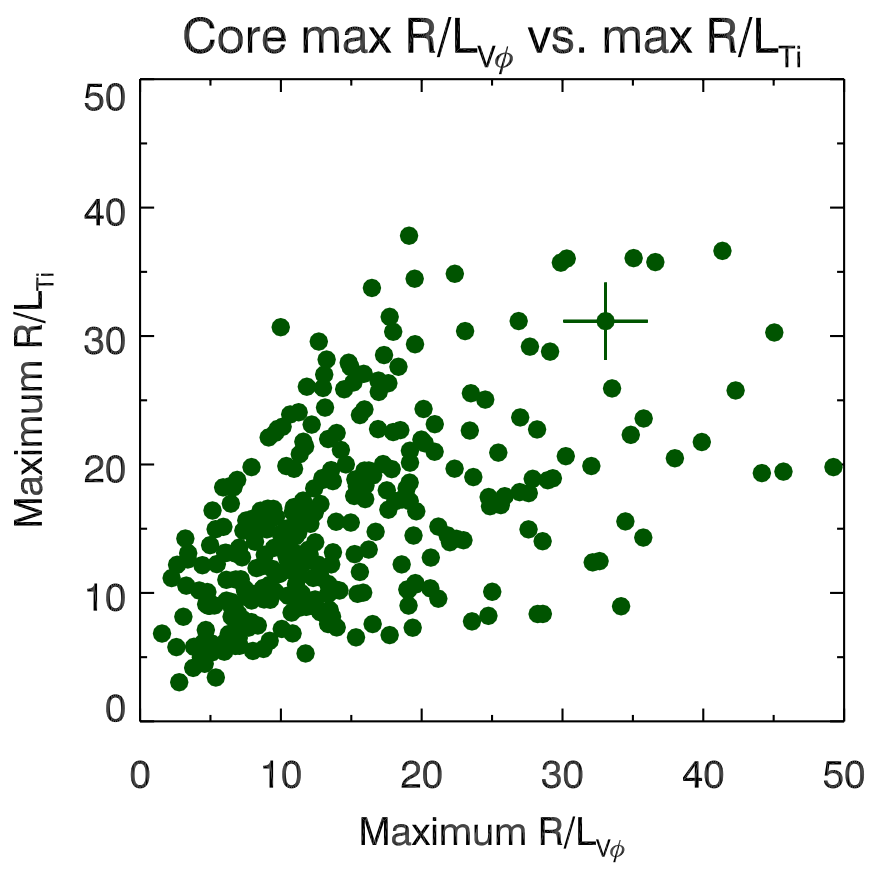

FIG. 6. (Color online) Peak normalized inverse ITG scale lengths $R / L_{T_{i}}$ vs peak normalized inverse velocity gradient scale lengths $R / L_{v_{\phi}}$.
Location of $\max \mathrm{E} \times \mathrm{B}$ shear and $\mathrm{i}-\mathrm{ITB}$

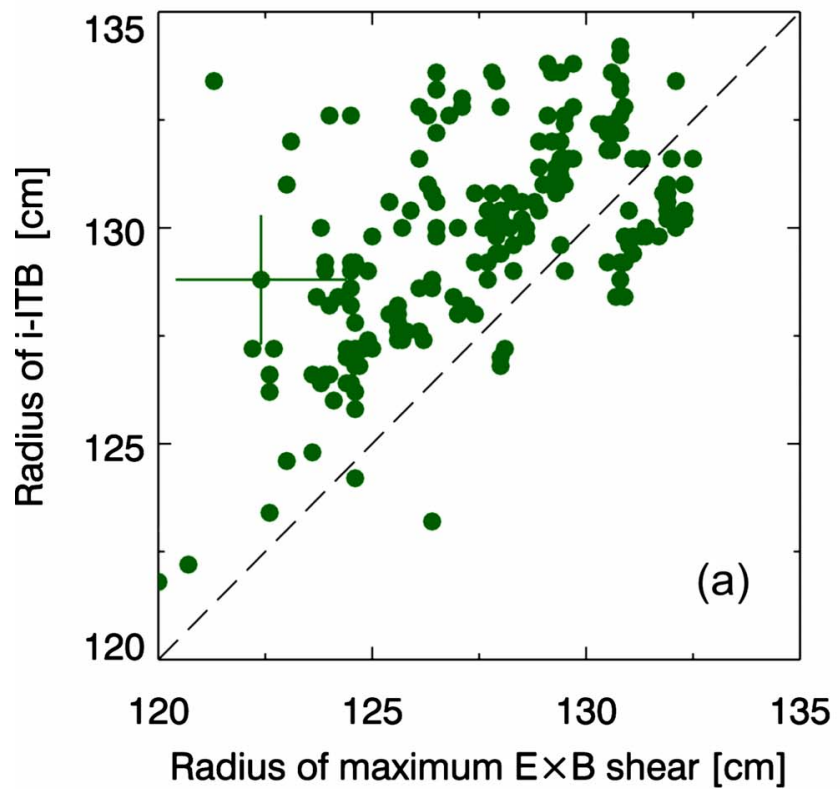

Radial separation between

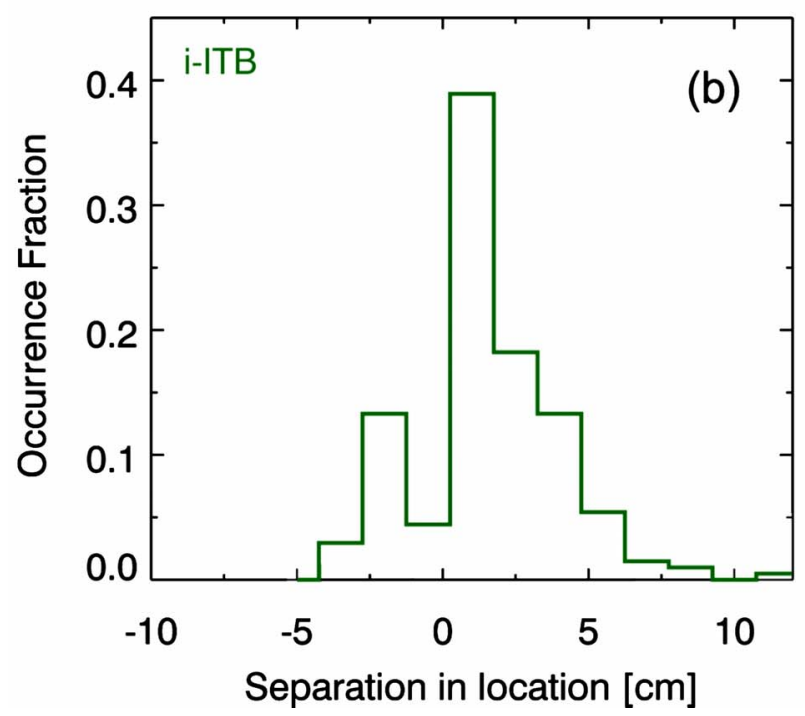

FIG. 7. (Color online) (a) Radius of the i-ITB compared to the location of maximum $\mathbf{E} \times \mathbf{B}$ shear, shown with equality line. (b) Histogram of radial separation between i-ITB and location of maxium $\mathbf{E} \times \mathbf{B}$ positive values indicate i-ITB is radially outwards.

location of the peak of the $\mathbf{E} \times \mathbf{B}$ shearing rate. These results are consistent with the general understanding that $\mathbf{E} \times \mathbf{B}$ shear can effectively suppress ITG induced ion turbulence, but that magnetic shear plays a more important role in electron transport suppression.

The transition out of the stiff $T_{e}$ profile regime due to sufficiently negative magnetic shear occurs at approximately $s_{\min } \leqslant-0.4$, as shown in Fig. 9, where the peak core $R / L_{T_{e}}$ is plotted against $s_{\min }$ for a large number of profiles. A comparison of profiles with $2 \mathrm{MW}$ NBI heating, shown as black circles, to profiles with the same NBI power and additional 


\section{Minimum magnetic shear and e-ITB locations}

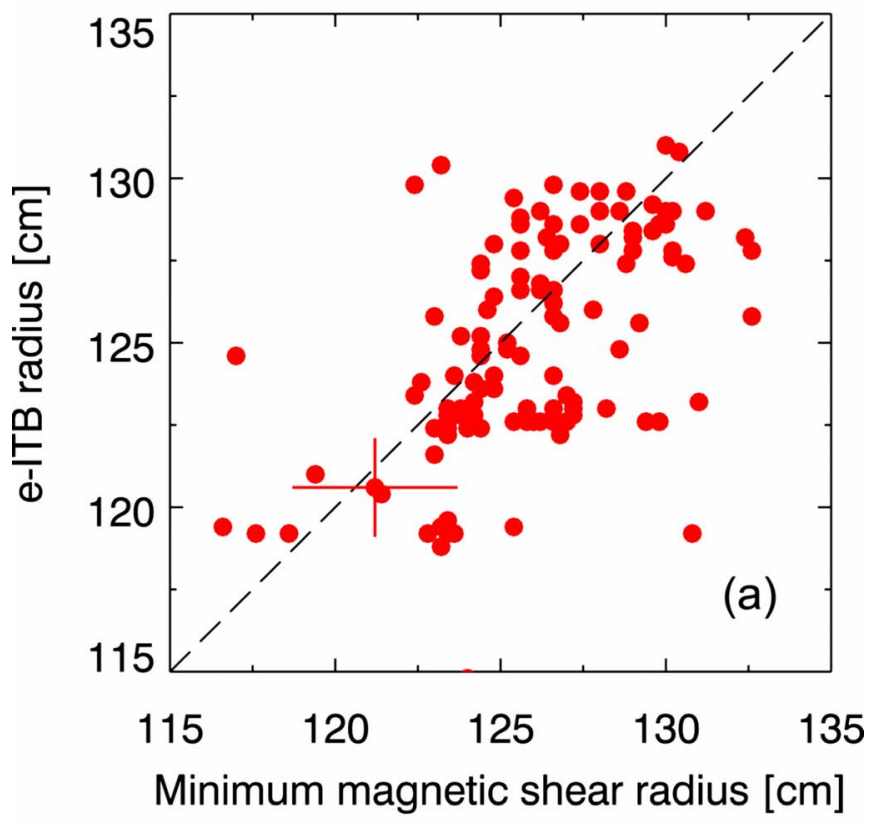

Radial separation between mag. shear minimum and peak core gradient

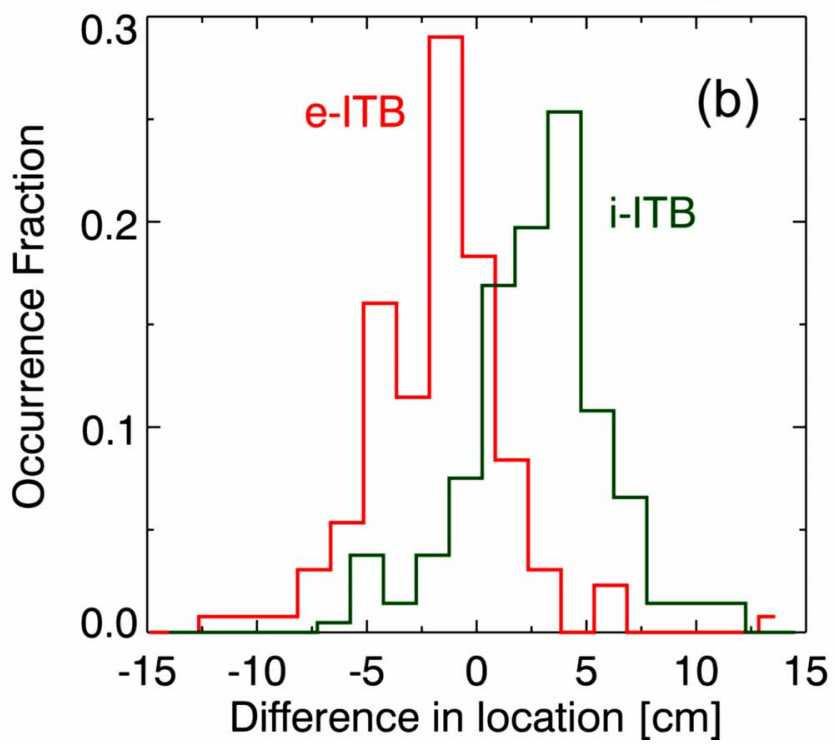

FIG. 8. (Color online) (a) Radius of the e-ITB vs radius of $s_{\min }$. (b) Histogram of radial separation between $s_{\min }$ and the e-ITB and i-ITBs. Positive values indicate ITB is radially outwards from $s_{\min }$.

HHFW power of up to $2 \mathrm{MW}$, shown as red squares, shows that $R / L_{T_{e}}$ only increases modestly with additional input power for $s_{\min } \gtrsim-0.4$, but can reach significantly higher values for more negative magnetic shear values. The inability to access high $R / L_{T_{e}}$ at zero or weakly reversed magnetic shear is emphasized by the shaded upper left region in Fig. 9, showing that for $s_{\min } \gtrsim-0.4$, peak $T_{e}$ gradients are limited to $R / L_{T_{e}} \lesssim 11$. The data also suggest that in the intermediate shear region, $-0.4 \geq s_{\min } \geq-1.0$, the maximum sustainable

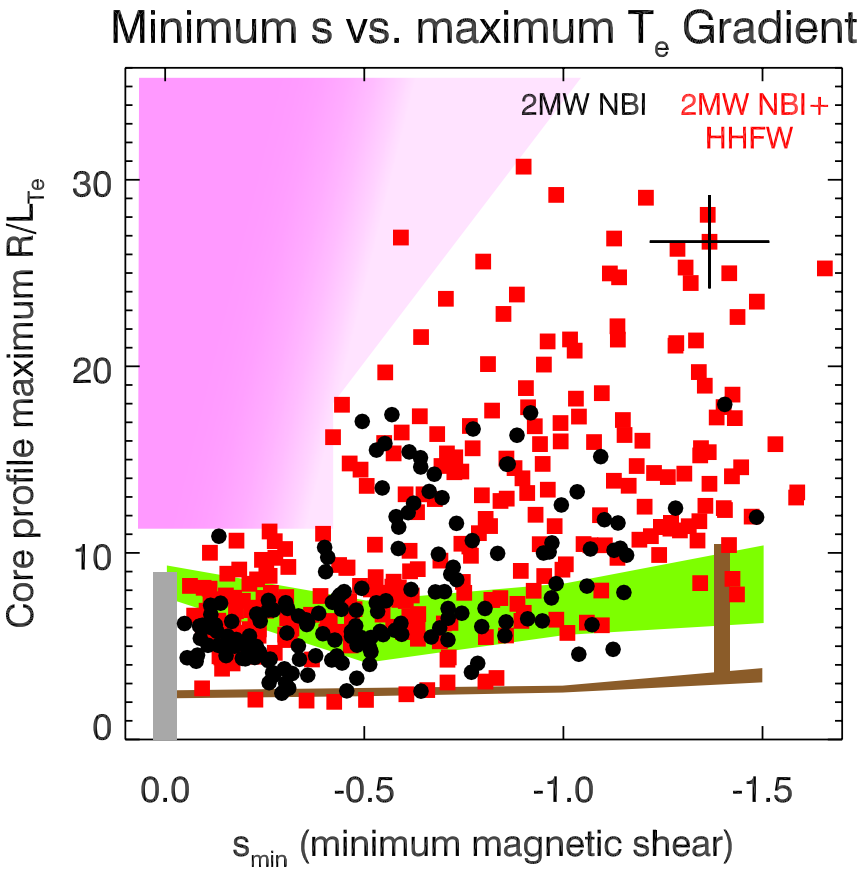

FIG. 9. (Color online) Value of minimum magnetic shear vs peak electron temperature gradient. Shaded region in the upper left shows an inaccessible region due to stiff profiles. Horizontal bands show critical ETG gradients calculated using linear GS2 and GYRO simulations. Gray vertical band at $s_{\text {min }}=0$ serve as a reference from high $\beta H$-mode discharges.

gradient may be a function of $s_{\min }$, but more data points at higher input power would be needed to confirm this.

The stiffness of the core $T_{e}$ profile is also observed in $H$-mode. Because NSTX $H$-modes have exclusively monotonic $q$-profiles with $s_{\min }=0$, these profiles are represented by the vertical bar at $s_{\min }=0$ in Fig. 9. A large number of $H$-mode profiles are included in the vertical band, including NBI powers up to $6 \mathrm{MW}$. The results show that stiff core $T_{e}$ profiles occur in both $H$-mode and $L$-mode discharges.

Although the data shown in Fig. 9 span a range of plasma parameters, no parameter other than magnetic shear was found to correlate well to $T_{e}$ profile stiffness. Plasma density at the e-ITB ranged from 1 to $4 \times 10^{19} \mathrm{~m}^{-3}$. $Z_{\text {eff }}$ ranged from 1.1 to 4 due to the use of both deuterium and helium as the bulk plasma species and varying wall conditions. Various rates of lithium evaporation ${ }^{16}$ were used during the experiment, primarily to control densities near the $\mathrm{rf}$ antennas to optimize coupling. The ratio of $T_{e} / T_{i}$ ranged from 0.4 to 2.5 at the e-ITB location. While critical ETG gradients are affected by terms such as $\tau=Z_{\text {eff }}\left(T_{e} / T_{i}\right)$, there was little correlation between this quantity and observed peak $R / L_{T_{e}}$. Horizontal bands in Fig. 9 show the critical gradients for ETG instability for a sample of profiles will be discussed in the section comparing measurements to simulations.

The combined effect of simultaneous electron and ion ITBs is shown in Fig. 10, showing ion thermal diffusivities near the neoclassical value at the i-ITB. Electron thermal diffusivities below that of the ions are typically not observed 


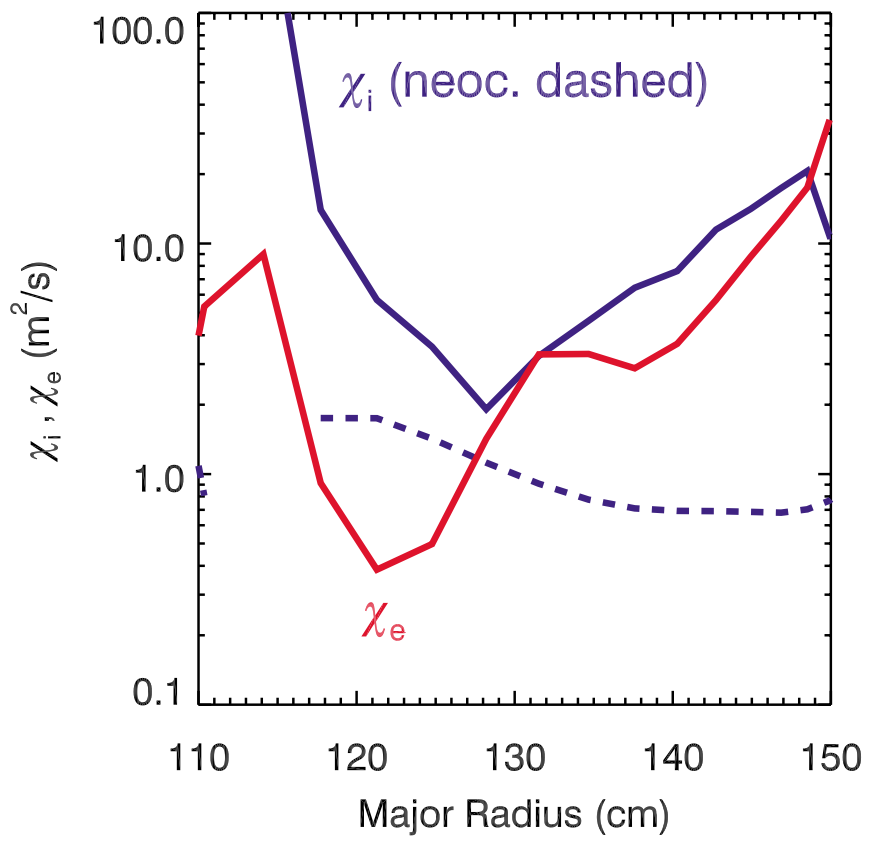

FIG. 10. (Color online) TRANSP calculations of the electron and ion thermal diffusivities with an ITB. Magnetic axis (not shown) is at $102 \mathrm{~cm}$ and the plasma edge is at $154 \mathrm{~cm}$.

without a strong e-ITB, with typical $\chi_{e}$ values observed in weakly reversed shear discharges ranging between $10-20 \mathrm{~m}^{2} / \mathrm{s}^{7}$

\section{ELECTRON FLUCTUATION MEASUREMENTS}

Having established a connection between negative magnetic shear and the ability to sustain higher electron temperature gradients, a microwave scattering diagnostic was used to measure local electron density fluctuations at the e-ITB at electron scale wave numbers $\left(k_{\perp} \rho_{e} \leq 0.6\right) .{ }^{17-19}$

Figure 11(a) compares fluctuation power spectra between two discharges, one with a weakly reversed shear $q$-profile without an e-ITB and another with a strongly reversed shear $q$-profile with an e-ITB. The $q$ and $T_{e}$ profiles corresponding to the fluctuation measurements are shown in Figs. 11(b) and 11(c). The high- $k$ fluctuations for the negative magnetic shear case are lower in amplitude over a wide frequency range despite elevated electron temperature gradients $\left(R / L_{T_{e}} \geq 20\right)$.

Fluctuation measurements were also taken outside the region of negative magnetic shear, near the location of $q_{\mathrm{min}}$, where the magnetic shear is zero. Measurements at this location did not display the same suppression of fluctuation regardless of the value of magnetic shear further inside the plasma, illustrating that turbulence suppression is a local phenomenon tied to the local magnetic shear value.

Examples of e-ITBs up until this point have used NBI for both heating and diagnostic purposes. Despite evidence showing that magnetic shear is the more important factor for electron transport, $\mathbf{E} \times \mathbf{B}$ shear has nonetheless been present. Figure 12(a) shows a comparison of high- $k$ fluctuation spectra between two discharges heated only with HHFW, using $50 \mathrm{~ms}$ NBI blips for diagnosis. The electron temperature pro-
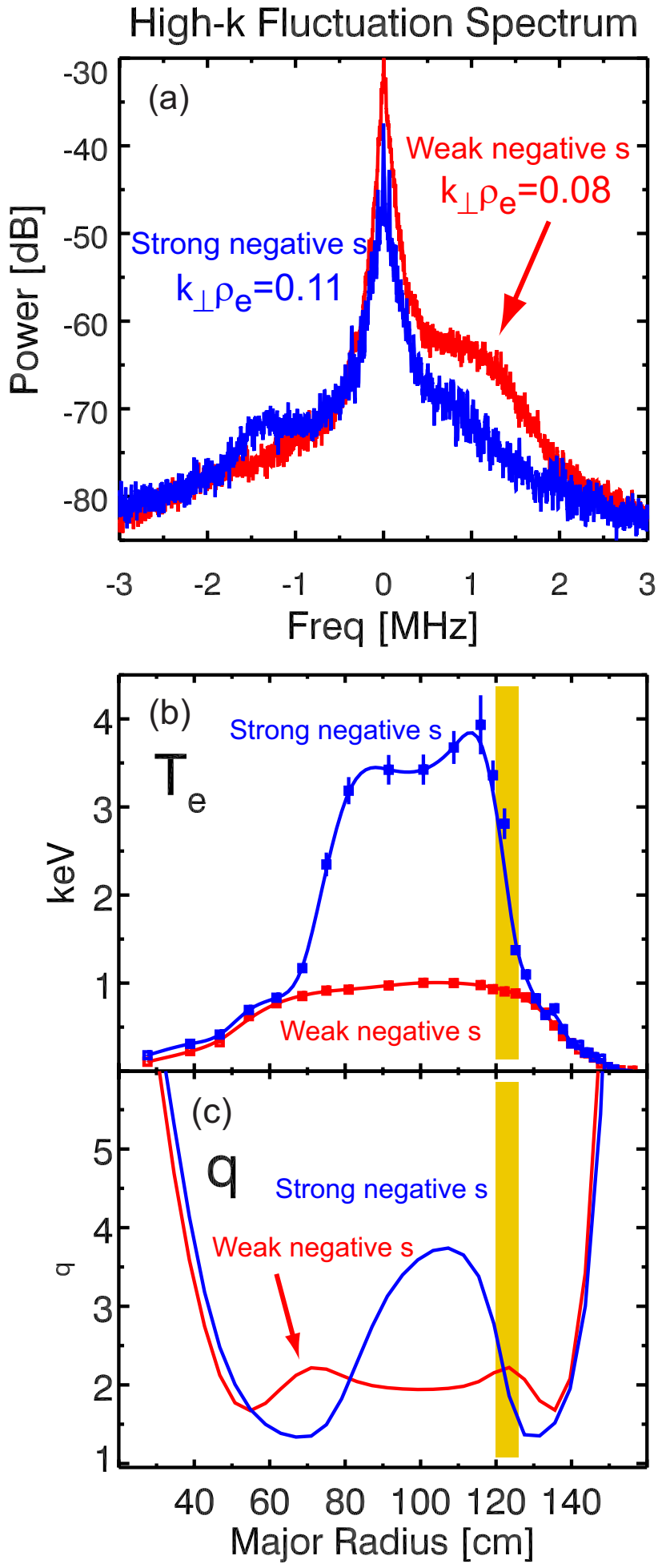

FIG. 11. (Color online) (a) High- $k$ microwave scattering fluctuation power spectra comparison between a case with an e-ITB and strongly negative magnetic shear vs a weakly reversed shear case with lower electron temperature gradients. (b) Electron temperatures and (c) $q$-profiles for cases shown in (a), shaded region indicates the high- $k$ measurement region.

files and $q$-profiles are shown in Figs. 12(b) and 12(c), with similar results as beam heated e-ITBs, where the strongly reversed shear discharge with steep $T_{e}$ gradients shows reduced fluctuations. $E_{r}$ and the $\mathbf{E} \times \mathbf{B}$ shearing rate for the e-ITB case are shown in Fig. 13. The lack of $\mathbf{E} \times \mathbf{B}$ shear around the fluctuation measurement region shows that mag- 

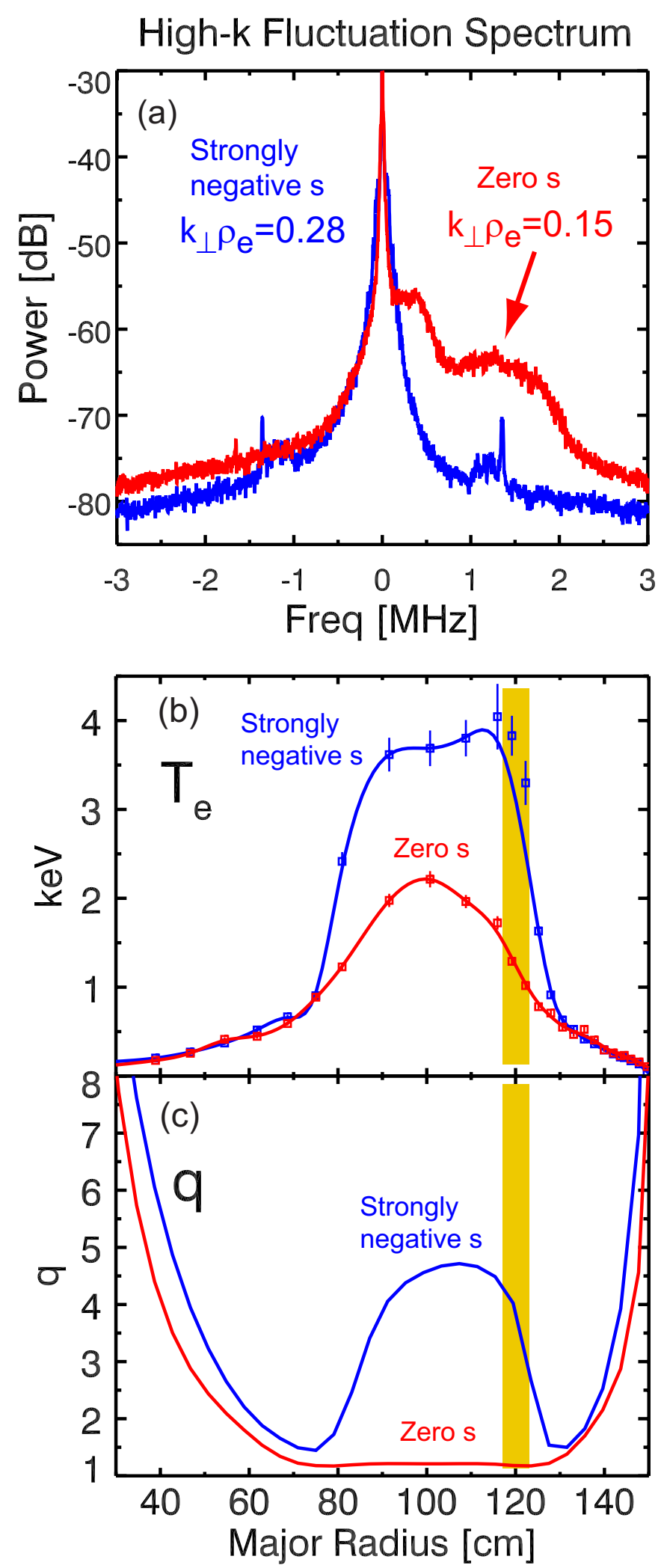

FIG. 12. (Color online) (a) High- $k$ fluctuation power spectra comparing two rf heated cases, a reversed shear e-ITB case, and a zero shear case. (b) Electron temperatures and (c) $q$-profiles for cases shown in (a), shaded region indicates the high- $k$ measurement region.

netic shear alone is capable of suppressing electron scale fluctuations. This calculation also excludes the poloidal velocity, which is expected to contribute minimally to the $\mathbf{E} \times \mathbf{B}$ shear in the plasma core.

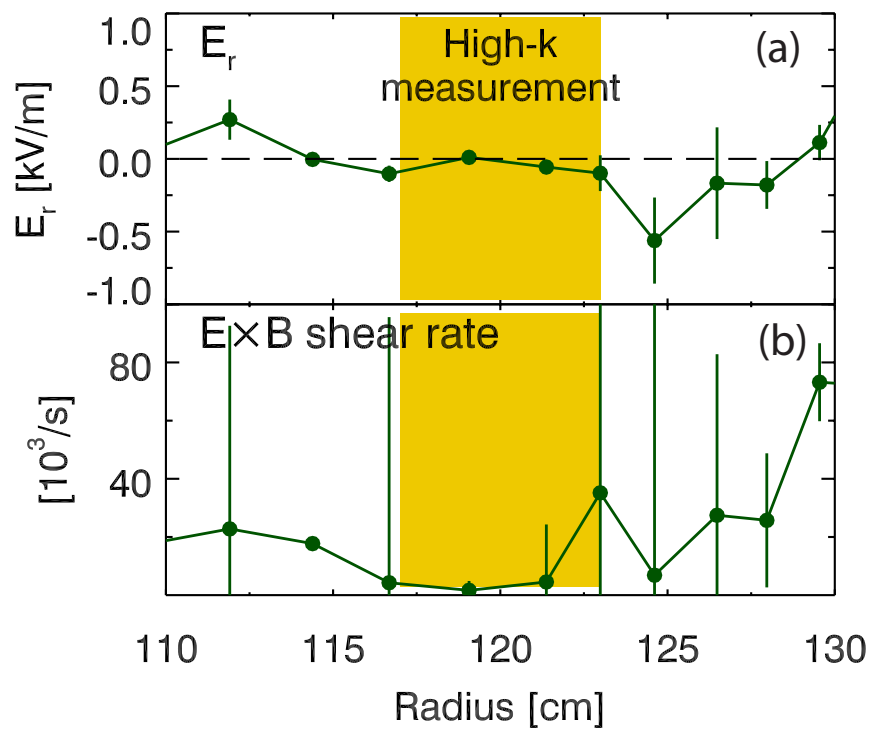

FIG. 13. (Color online) (a) $E_{r}$ at the high- $k$ measurement region (b) $\mathbf{E} \times \mathbf{B}$ shearing rate at the high- $k$ measurement region.

Fluctuation power was statistically analyzed and is shown in Fig. 14 where the fluctuation power was integrated over frequency for $5 \mathrm{~ms}$ periods immediately preceding each kinetic profile for two high- $k$ channels. Due to local $T_{e}$ variation at the measurement location, not every measurement point is identical in $k_{\perp} \rho_{e}$. However, the fact that both high- $k$ channels at different wave numbers show similar results suggests that fluctuations are suppressed over a range of wave numbers. While a large range of fluctuation power exists for the zero or weakly reversed sheared plasmas, this could be explained by variations in critical gradients for electron gyroscale modes due to varying plasma parameters. A trend can be seen toward increasing fluctuation power with increasing electron temperature gradients, though with significant scatter. What is clear, however, is that strongly reversed sheared e-ITBs display lower levels of integrated fluctuation power, by about an order of magnitude, across a range of $k_{\perp} \rho_{e}$ despite having significantly higher electron temperature gradients.

\section{COMPARISON TO GYROKINETIC SIMULATIONS}

Linear gyrokinetic simulations were used to calculate critical temperature gradients, $\left(R / L_{T_{e}}\right)_{\text {crit }}$, for the onset of ETG modes over the range of measured magnetic shear and electron temperature gradients. For GS2 simulations, ${ }^{20}$ critical gradients were calculated for sample experimental profiles with and without ITBs. The calculated range is shown by the larger shaded band (green) in Fig. 9. The narrower bands (brown) in the same figure show results from linear GYRO simulations, ${ }^{15,21}$ where a single ITB profile was used but the parameters $\tau=Z_{\mathrm{eff}} T_{e} / T_{i}$ and the magnetic shear were varied in the simulations to account for errors and variations observed in the experiments. Because the ETG $\left(R / L_{T}\right)_{\text {crit }}$ does not change significantly with increasingly negative magnetic shear, these results show that the measured electron temperature gradient can greatly exceed critical ETG gradients, but only for strongly negative magnetic shear. 


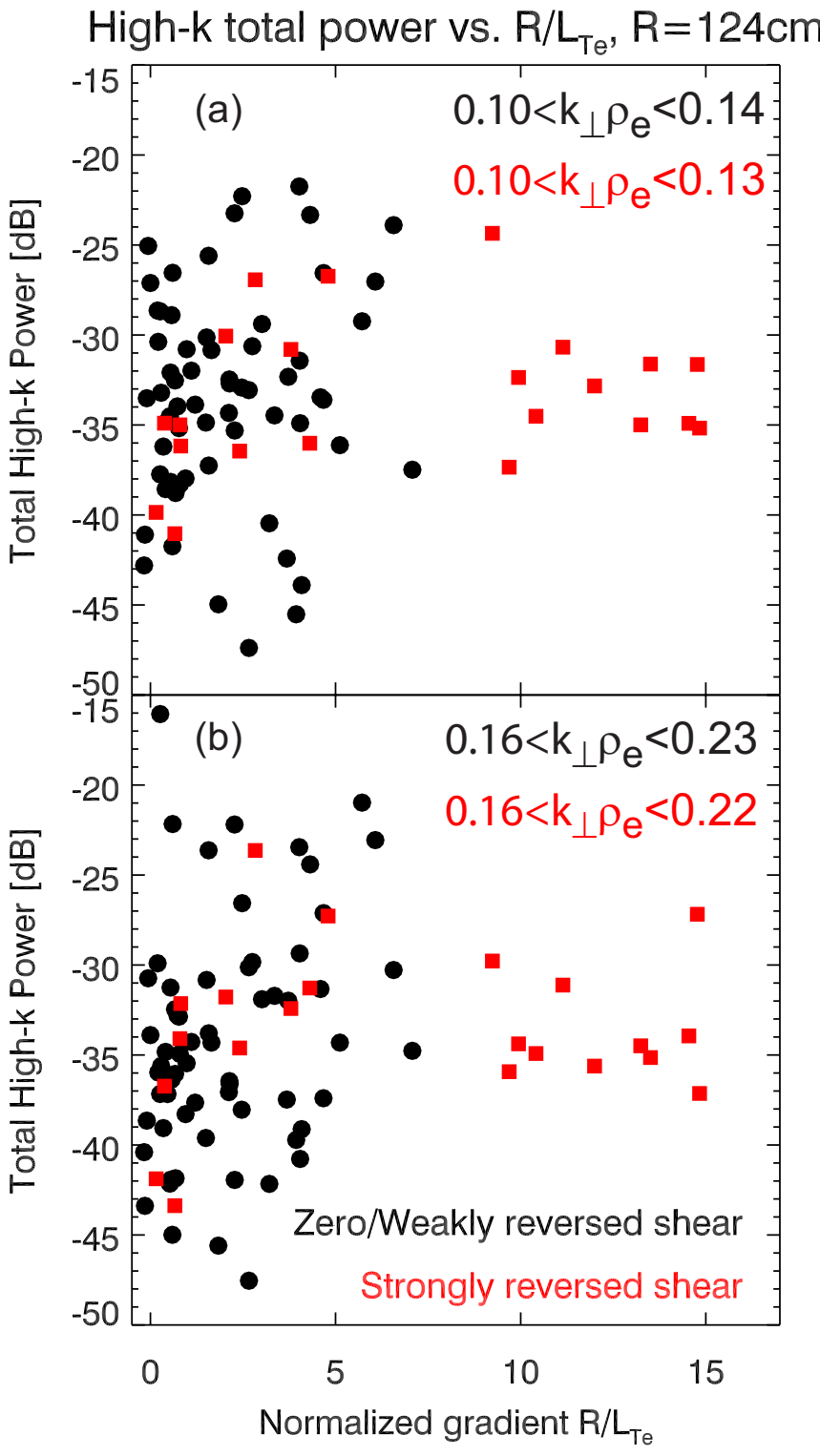

FIG. 14. (Color online) Integrated high- $k$ power statistics at two ranges of $k_{\perp} \rho_{e}$ vs the local $R / L_{T_{e}}$ at the fluctuation measurement location. $k_{\perp} \rho_{e}$ variation is due to changes in $T_{e}$ at the measurement location.

The connection between high- $k$ fluctuations on NSTX and ETG microinstabilities has been previously established in Ref. 22 and the currently observed reduction in fluctuation levels are consistent with previous works from Ref. 23, where nonlinear gyrokinetic simulation results showed that electron thermal transport due to ETG modes would be significantly reduced at strongly negative magnetic shears.

\section{CONCLUSION}

In conclusion, we observed internal transport barriers in NSTX for reversed shear discharges in the electron thermal, ion thermal, and toroidal momentum channels. Statistical analysis of a kinetic profile database shows that the i-ITB is best correlated with the location of maximum $\mathbf{E} \times \mathbf{B}$ shear and to the value of the toroidal velocity shear. The e-ITB, on the other hand, is best correlated with $s_{\min }$ and can show a transition out of a stiff profile regime when the magnetic shear reaches a sufficiently negative value. Density fluctuation measurements at the electron gyroscale in the steep gradient region of the e-ITB show reduced fluctuation levels despite greatly exceeding the ETG $\left(R / L_{T_{e}}\right)_{\text {crit }}$ relative to zero and weakly sheared profiles. The combination of e-ITB and i-ITB results in significantly reduced thermal diffusivities.

\section{ACKNOWLEDGMENTS}

We are grateful for the support and hard work from the NSTX Team and PPPL. This research is supported by US DOE Grant No. DE-FG02-99ER54520 and US DOE Contract No. DE-AC02-76CH03073.

${ }^{1}$ J. Connor, T. Fukuda, X. Garbet, C. Gormezano, V. Mukhovatov, and M. Wakatani, the ITB Database Group, the ITPA Topical Group on Transport, and IB Physics, Nucl. Fusion 44, R1 (2004).

${ }^{2}$ H. Meyer, A. R. Field, R. J. Akers, C. Brickley, N. J. Conway, A. Patel, P. G. Carolan, C. Challis, G. F. Counsell, G. Cunningham, P. Helander, A. Kirk, B. Lloyd, R. Maingi, M. R. Tournianski, M. J. Walsh, and MAST and NBI Teams, Plasma Phys. Controlled Fusion 46, A291 (2004).

${ }^{3}$ J. R. Wilson, R. E. Bell, S. Bernabei, M. Bitter, P. Bonoli, D. Gates, J. Hosea, B. LeBlanc, T. K. Mau, S. Medley, J. Menard, D. Mueller, M. Ono, C. K. Phillips, R. I. Pinsker, R. Raman, A. Rosenberg, P. Ryan, S. Sabbagh, D. Stutman, D. Swain, Y. Takase, J. Wilgen, and NSTX Team, Phys. Plasmas 10, 1733 (2003).

${ }^{4}$ J. Hosea, R. E. Bell, B. P. LeBlanc, C. K. Phillips, G. Taylor, E. Valeo, J. R. Wilson, E. F. Jaeger, P. M. Ryan, J. Wilgen, H. Yuh, F. Levinton, S. Sabbagh, K. Tritz, J. Parker, P. T. Bonoli, R. Harvey, and NSTX Team, Phys. Plasmas 15, 056104 (2008).

${ }^{5}$ B. P. LeBlanc, R. E. Bell, D. W. Johnson, D. E. Hoffman, D. C. Long, and R. W. Palladino, Rev. Sci. Instrum. 74, 1659 (2003).

${ }^{6}$ R. Bell, D. Johnson, R. Feder, and T. Biewer, Bulletin of the 47th Annual Meeting of the Division of Plasma Physics of the American Physical Society, 2005 (unpublished), Vol. 50, p. RP1.005.

${ }^{7}$ F. M. Levinton, H. Yuh, M. G. Bell, R. E. Bell, L. Delgado-Aparicio, M. Finkenthal, E. D. Fredrickson, D. A. Gates, S. M. Kaye, B. P. LeBlanc, R. Maingi, J. E. Menard, D. Mikkelsen, D. Mueller, R. Raman, G. Rewoldt, S. A. Sabbagh, D. Stutman, K. Tritz, and W. Wang, Phys. Plasmas 14, 056119 (2007).

${ }^{8}$ B. LeBlanc and R. Bell, private communication (December, 2008).

${ }^{9}$ E. J. Synakowski, S. H. Batha, M. A. Beer, M. G. Bell, R. E. Bell, R. V. Budny, C. E. Bush, P. C. Efthimion, T. S. Hahm, G. W. Hammett, B. LeBlanc, F. Levinton, E. Mazzucato, H. Park, A. T. Ramsey, G. Schmidt, G. Rewoldt, S. D. Scott, G. Taylor, and M. C. Zarnstorff, Phys. Plasmas 4, 1736 (1997).

${ }^{10}$ K. H. Burrell, Phys. Plasmas 4, 1499 (1997).

${ }^{11}$ T. S. Hahm and K. H. Burrell, Phys. Plasmas 2, 1648 (1995).

${ }^{12}$ R. E. Waltz, R. L. Dewar, and X. Garbet, Phys. Plasmas 5, 1784 (1998).

${ }^{13}$ J. E. Kinsey, R. E. Waltz, and J. Candy, Phys. Plasmas 12, 062302 (2005).

${ }^{14}$ W. A. Houlberg, K. C. Shaing, S. P. Hirshman, and M. C. Zarnstorff, Phys. Plasmas 4, 3230 (1997).

${ }^{15}$ J. Peterson, G. Hammett, D. Mikkelsen, S. Kaye, J. Candy, and R. E. Waltz, Bulletin of the 50th Annual Meeting of the Division of Plasma Physics of the American Physical Society, 2008 (unpublished), Vol. 50, p. NP6.096.

${ }^{16}$ H. Kugel, M. Bell, R. Bell, C. Bush, D. Gates, T. Gray, R. Kaita, B. Leblanc, R. Maingi, R. Majeski, D. Mansfield, D. Mueller, S. Paul, R. Raman, A. L. Roquemore, S. Sabbagh, C. H. Skinner, V. Soukhanovskii, T. Stevenson, and L. Zakharov, J. Nucl. Mater. 363, 791 (2007).

${ }^{17}$ E. Mazzucato, Phys. Plasmas 10, 753 (2003).

${ }^{18}$ D. R. Smith, E. Mazzucato, T. Munsat, H. Park, D. Johnson, L. Lin, C. W. Domier, M. Johnson, and J. N. C. Luhmann, Rev. Sci. Instrum. 75, 3840 (2004).

${ }^{19}$ D. R. Smith, E. Mazzucato, W. Lee, H. K. Park, C. W. Domier, and J. N. C. Luhmann, Rev. Sci. Instrum. 79, 123501 (2008). 
${ }^{20}$ M. Kotschenreuther, G. Rewoldt, and W. M. Tang, Comput. Phys. Commun. 88, 128 (1995).

${ }^{21}$ J. Candy and R. E. Waltz, J. Comput. Phys. 186, 545 (2003).

${ }^{22}$ E. Mazzucato, D. R. Smith, R. E. Bell, S. M. Kaye, J. C. Hosea,
B. P. LeBlanc, J. R. Wilson, P. M. Ryan, C. W. Domier, N. C. Luhmann, Jr., H. Yuh, W. Lee, and H. Park, Phys. Rev. Lett. 101, 075001 (2008).

${ }^{23}$ F. Jenko and W. Dorland, Phys. Rev. Lett. 89, 225001 (2002). 